Pacific

Journal of

Mathematics

\title{
CHEBYSHEV PROPERTY \\ OF COMPLETE ELLIPTIC INTEGRALS \\ AND ITS APPLICATION TO ABELIAN INTEGRALS
}

Armengol Gasull, Weigu Li, Jaume Llibre, and Zhifen Zhang

Volume $202 \quad$ No. 2

February 2002 


\section{CHEBYSHEV PROPERTY \\ OF COMPLETE ELLIPTIC INTEGRALS AND ITS APPLICATION TO ABELIAN INTEGRALS}

\section{Armengol Gasull, Weigu Li, Jaume Llibre, and Zhifen Zhang}

This paper has two parts. In the first one we study the maximum number of zeros of a function of the form $f(k) K(k)+$ $g(k) E(k)$, where $k \in(-1,1), f$ and $g$ are polynomials, and $K(k)=\int_{0}^{\pi / 2} \frac{d \theta}{\sqrt{1-k^{2} \sin ^{2} \theta}}$ and $E(k)=\int_{0}^{\pi / 2} \sqrt{1-k^{2} \sin ^{2} \theta} d \theta$ are the complete normal elliptic integrals of the first and second kinds, respectively. In the second part we apply the first one to obtain an upper bound for the number of limit cycles which appear from a small polynomial perturbation of the planar isochronous differential equation $\dot{z}=i z+z^{3}$, where $z=x+i y \in \mathbb{C}$.

\section{Introduction and statement of the main results.}

In the qualitative theory of real planar differential systems the main open problem is the determination of limit cycles. A classical way to obtain limit cycles is perturbing the periodic orbits of a center. There are several methods for studying the bifurcated limit cycles from a center. The major part of the methods are based either on the Poincaré return map, or on the Poincaré-Melnikov integral or Abelian integral which are equivalent in the plane (see for instance [1]). Recently some other methods are presented, ones based on the inverse integrating factor (see [7]), others are based in the reduction of the problem to a one dimensional differential equation (see $[\mathbf{1 0}]$ and $[\mathbf{1 3}]$ ). In general these methods are difficult to apply for studying the limit cycles that bifurcate from the periodic orbits of a center when the system is integrable but not Hamiltonian. As far as we know few papers study the non-Hamiltonian centers, see for instance $[\mathbf{3}],[\mathbf{5}],[\mathbf{8}],[\mathbf{9}],[\mathbf{1 0}]$, [11] and [13].

By definition a polynomial system is a differential system of the form

$$
\frac{d x}{d t}=P(x, y), \quad \frac{d y}{d t}=Q(x, y),
$$

where $P$ and $Q$ are polynomials with real coefficients. We say that $n=$ $\max \{\operatorname{deg} P, \operatorname{deg} Q\}$ is the degree of the polynomial system. 
In $[\mathbf{1 1}]$ we studied the limit cycles that bifurcate from the periodic orbits of quadratic isochronous centers when we perturb these centers inside the class of all polynomial systems of degree $n$. The case $n=2$ was studied by Chicone and Jacobs $[\mathbf{3}]$.

The number of zeros of Abelian integrals for reversible isochronous cubic centers whose all orbits are conic were studied in [12].

The technique for studying the limit cycles that bifurcate from the periodic orbits of a integrable non-Hamiltonian center when we perturb the center is classical, see for instance [17]. If the perturbed system is

$$
\begin{aligned}
& \dot{x}=f(x, y)+\varepsilon P(x, y), \\
& \dot{y}=g(x, y)+\varepsilon Q(x, y),
\end{aligned}
$$

and $1 / R(x, y)$ is an integrating factor when $\varepsilon=0$, then the number of zeros of the following Abelian integral

$$
M(h):=\int_{\{H(x, y)=h\}} \frac{P(x, y) d x+Q(x, y) d y}{R(x, y)},
$$

where $H(x, y)$ is such that $\frac{\partial H(x, y)}{\partial x}=\frac{g(x, y)}{R(x, y)},-\frac{\partial H(x, y)}{\partial y}=\frac{g(x, y)}{R(x, y)}$, and $\{H(x, y)$ $=h\}$ are periodic orbits of the unperturbed system, controls the number of limit cycles of the perturbed system for $\varepsilon$ small enough.

When we calculate an Abelian integral we often meet elliptic integrals which can be expressed as the following complete normal elliptic integrals of the first and second kinds:

$$
K(k)=\int_{0}^{\pi / 2} \frac{d \theta}{\sqrt{1-k^{2} \sin ^{2} \theta}}, \quad E(k)=\int_{0}^{\pi / 2} \sqrt{1-k^{2} \sin ^{2} \theta} d \theta .
$$

Therefore to estimate the number of zeros of a function of the form

$$
f(k) K(k)+g(k) E(k),
$$

where $f, g$ are real polynomials of $k$, is important. In order to get a good estimation we need to prove a kind of Chebyshev property for (3). This is the goal of the first part of this paper.

We denote by $\mathcal{P}_{n}$ the set of all real polynomials in one variable of degree at most $n$. The next two theorems are the main results of this paper.

Theorem 1. For $f \in \mathcal{P}_{n}, g \in \mathcal{P}_{m}$ and $k \in(-1,1)$ an upper bound for the number of zeros of the function $M(k)=f(k) K(k)+g(k) E(k)$, taking into account their multiplicities, is $n+m+2$. Moreover:

(a) For arbitrary $k_{i} \in(-1,1)$ with $i=1,2, \ldots, n+m+1$ there exist $f \in \mathcal{P}_{n}$ and $g \in \mathcal{P}_{m}$ such that $M(k) \not \equiv 0$ and $M\left(k_{i}\right)=0$ for $i=$ $1,2, \ldots, n+m+1$.

(b) There exist values of $n$ and $m$ (for instance, both values even) for which the upper bound $n+m+2$ is attained. 
(c) There exist values of $n$ and $m$ (for instance, $n<m+2$ and $n+m$ odd) for which the upper bound is $n+m+1$.

Theorem 1 will be proved in Section 2 by using the Argument Principle. As far as we know this method to estimate the number of zeros of Abelian integrals was introduced by Petrov in $[\mathbf{1 4}, \mathbf{1 5}, \mathbf{1 6}]$.

As an application of Theorem 1, in the second part of this paper we study the number of limit cycles which bifurcate from the closed curves surrounding the origin of the planar holomorphic isochronous center, $\dot{z}=$ $i z+z^{3}$. In other words, we study the number of zeros of the Abelian integral associated to the system

$$
\dot{z}=i z+z^{3}+\varepsilon P(z, \bar{z}),
$$

where $P$ is a polynomial of degree $n$.

Theorem 2. An upper bound for the number of zeros (taking into account their multiplicitis) of the Abelian integral associated to system (4) for $n \geq 9$ is $3 n-1$.

Theorem 2 will be proved in Section 3 . The cases $n<9$ also would follow from a more accurate analysis of the computations made in this paper, but we are just interested into obtaining an asymptotic bound.

Note that, as in most known examples, the upper bound for the number of zeros of the Abelian integrals associated to the perturbed system depends linearly on the degree of the polynomial perturbation.

\section{Elliptic functions.}

Before starting the proof we need several preliminary results on the elliptic functions $K(k)$ and $E(k)$ and their extensions to the complex plane.

Lemma 3 ([2, formulas 118.02-710.00]). The functions $K$ and $E$ satisfy the following Picard-Fuchs equation:

$$
\frac{d K}{d k}=\frac{E-\left(1-k^{2}\right) K}{k\left(1-k^{2}\right)}, \quad \frac{d E}{d k}=\frac{E-K}{k},
$$

and the following linear differential equations of order two:

$$
\begin{aligned}
& k\left(1-k^{2}\right) \frac{d^{2} K}{d k^{2}}+\left(1-3 k^{2}\right) \frac{d K}{d k}-k K=0, \\
& k\left(1-k^{2}\right) \frac{d^{2} E}{d k^{2}}+\left(1-k^{2}\right) \frac{d E}{d k}+k E=0 .
\end{aligned}
$$

From (5) and since $K$ and $E$ are clearly analytic at $k=0$, the elliptic integrals $E$ and $K$ can be continuously extended to single-valued analytic functions on the region

$$
D=\mathbb{C} \backslash\{z \in \mathbb{R},|z| \geq 1\} .
$$


Denote the upper and lower banks of the cut $\{z \in \mathbb{R}, z \geq 1\}$ by $L_{+}^{1}$ and $L_{+}^{2}$ respectively; and the upper and lower banks of the cut $\{z \in \mathbb{R}, z \leq-1\}$ by $L_{-}^{1}$ and $L_{-}^{2}$, respectively. Next lemma collects several properties of these extended functions.

Lemma 4. Consider the extensions of $K$ and $E$ to $D \subset \mathbb{C}$. As usual,we denote by $\log$ the principal determination of the logarithm function. They satisfy the following properties:

(a) The asymptotic expansions of $K$ and $E$ near \pm 1 are given by

$$
\begin{aligned}
K= & \log 4-\frac{1}{2} \log \left(1-k^{2}\right)+O\left(\left|\left(\log \left(1-k^{2}\right)\right)\left(1-k^{2}\right)\right|\right), \\
E= & 1+\frac{1}{2}\left[\log 4-\frac{1}{2} \log \left(1-k^{2}\right)-\frac{1}{2}\right]\left(1-k^{2}\right) \\
& +O\left(\left|\left(\log \left(1-k^{2}\right)\right)\left(1-k^{2}\right)^{2}\right|\right) .
\end{aligned}
$$

(b) The asymptotic expansions of $K$ and $E$ near $\infty$ are given by

$$
K \sim k^{-1} \log k, \quad E \sim k .
$$

(c) For $k \in L_{ \pm}^{i}, i=1,2$ the following hold

$$
K E \neq 0, \quad \operatorname{Im}\left(\frac{K}{E}\right) \neq 0, \quad \operatorname{Im}\left(\frac{E}{K}\right) \neq 0 .
$$

(d) For $k \in L_{ \pm}^{i}, i=1,2$ the following holds

$$
(\operatorname{Im} K)(\operatorname{Im} E) \neq 0 .
$$

Proof. (a). These expressions are given in formulas 900.05 and 900.10 of [2].

(b). By introducing the new variable $t=1 / k$, the differential equation for $K$ in (6) is changed into

$$
t^{2} \frac{d^{2} K}{d t^{2}}+t \frac{t^{2}+1}{t^{2}-1} \frac{d K}{d t}+\frac{1}{1-t^{2}} K=0 .
$$

The indicial equation associated with $(7)$ is $\lambda(\lambda-1)-\lambda+1=0$, which has the double root $\lambda=1$. By applying the Frobenius method (see for instance [4, pp. 132-135]), we know that Equation (7) has two independent solutions of the form

$$
\varphi_{1}(t)=t \sum_{j=0}^{\infty} c_{j} t^{j}, \quad c_{0}=1
$$

and

$$
\varphi_{2}(t)=\varphi_{1}(t) \log t+\sum_{j=2}^{\infty} d_{j} t^{j}
$$


Therefore

$$
K(k)=a_{1} \varphi_{1}\left(k^{-1}\right)+a_{2}\left[-\varphi_{1}\left(k^{-1}\right) \log k+\sum_{j=2}^{\infty} d_{j} k^{-j}\right],
$$

for some constants $a_{1}$ and $a_{2}$. Now we prove that $a_{2} \neq 0$. Consider $k=$ $i s, s \in \mathbb{R}^{+}$. Then note that

$$
\begin{aligned}
|k K(k)| & =\left|i \int_{0}^{\pi / 2} \frac{s}{\sqrt{1+s^{2} \sin ^{2} \theta}} d \theta\right| \\
& =\int_{0}^{\pi / 2} \frac{s}{\sqrt{1+s^{2} \sin ^{2} \theta}} d \theta \geq \int_{0}^{\pi / 2} \frac{s}{\sqrt{1+s^{2} \theta^{2}}} d \theta \\
& =\int_{0}^{s \pi / 2} \frac{d t}{\sqrt{1+t^{2}}} \stackrel{s \rightarrow \infty}{\longrightarrow} \infty .
\end{aligned}
$$

Hence $a_{2} \neq 0$ and we have proved that $K(k) \sim k^{-1} \log k$.

The proof that $E(k) \sim k$ follows the same steps.

(c). All the results of this statement will follow if we prove that

$$
f(k):=(\operatorname{Re} K)(\operatorname{Im} E)-(\operatorname{Re} E)(\operatorname{Im} K)
$$

does not vanish on $k \in L_{ \pm}^{i}, i=1,2$. In fact we will prove that

$$
f(k)= \pm(-1)^{i} \frac{\pi}{2} \neq 0, \quad k \in L_{ \pm}^{i}, \quad i=1,2 .
$$

Note that for $k \in L_{ \pm}^{i}$, the vector $(\operatorname{Re} K, \operatorname{Re} E)$ and $(\operatorname{Im} K, \operatorname{Im} E)$ are the solutions of (5). Hence by Liouville's Formula

$$
\frac{d f}{d k}=\operatorname{tr}\left(\begin{array}{cc}
-\frac{1}{k} & \frac{1}{k\left(1-k^{2}\right)} \\
-\frac{1}{k} & \frac{1}{k}
\end{array}\right) f=0,
$$

which implies that $f$ is a constant. On the other hand, by statement (a), we have

$$
\begin{aligned}
f(k)= & \left(\log 4-\frac{1}{2} \log \left|1-k^{2}\right|+o\left(\left|1-k^{2}\right|^{\frac{1}{2}}\right)\right)\left(O\left(\left|1-k^{2}\right|\right)\right) \\
& -\left(1+o\left(\left|1-k^{2}\right|^{\frac{1}{2}}\right)\right)\left(\mp(-1)^{i} \frac{\pi}{2}+o\left(\left|1-k^{2}\right|\right)\right), \quad k \in L_{ \pm}^{i} .
\end{aligned}
$$

Let $k \rightarrow \pm 1$, we get $f= \pm(-1)^{i} \frac{\pi}{2}, k \in L_{ \pm}^{i}, i=1,2$, so statement (c) is proved. 


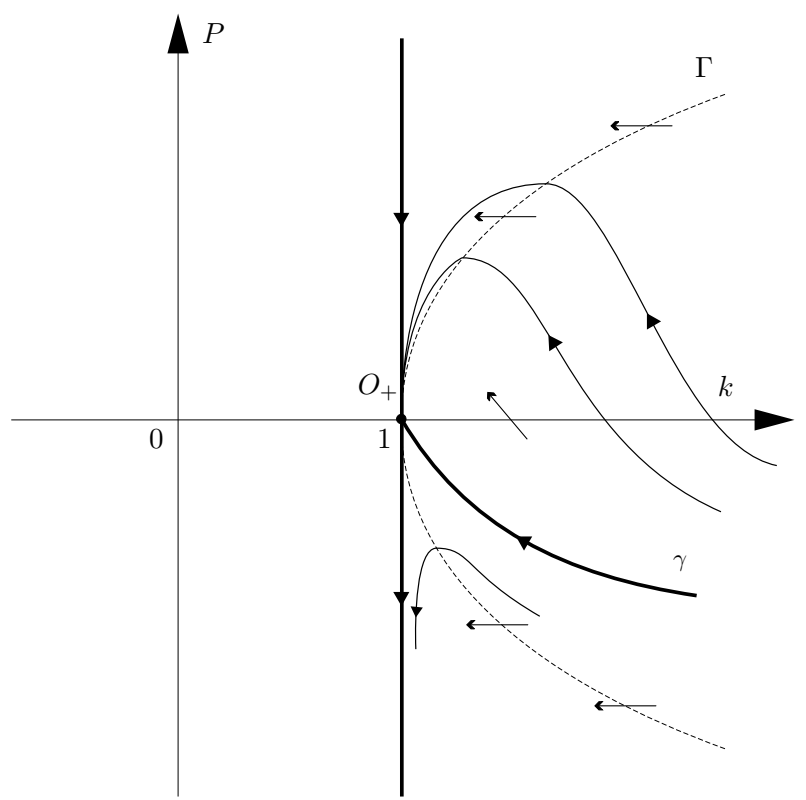

Figure 1. Phase portrait of differential equation (8) near $O_{+}=(1,0)$.

(d). Remember that on the banks $L_{ \pm}^{i}$, the vector $(\operatorname{Im} K, \operatorname{Im} E)$ is a solution of (1). Therefore the function $P=\operatorname{Im} E / \operatorname{Im} K$ satisfies the following Ricatti equation:

$$
\frac{d P}{d k}=\frac{-P^{2}+2\left(1-k^{2}\right) P+k^{2}-1}{k\left(1-k^{2}\right)},
$$

or its equivalent system

$$
\begin{aligned}
& \frac{d k}{d t}=k\left(1-k^{2}\right), \\
& \frac{d P}{d t}=-P^{2}+2\left(1-k^{2}\right) P+k^{2}-1 .
\end{aligned}
$$

We will only prove the result when $k \in L_{+}^{i}$, the case $k \in L_{-}^{i}$ can be proved in a similar way.

The phase portrait of $(8)$ is given in Figure 1. Note that by Lemma 4.(a), $\lim _{k \rightarrow 1} P(k)=0$, so $P(k)$ belongs to the stable set of the saddle-node $O_{+}=$ $(1,0)$. All orbits except the stable separatrix $\gamma$ in the stable set of $O_{+}$tend to $O_{+}$in the tangential direction along the half line $k=1, P \geq 0$. On the other hand, again by Lemma 4.(a), $|\operatorname{Im} E / \operatorname{Im} K| \sim 1-k$, at $k=1$. Therefore $P(k)$ must be the stable manifold of $O_{+}$, which is located between the horizontal isocline $\Gamma$ and $x$-axis, as shown in Figure 1 . Hence $(\operatorname{Im} E)(\operatorname{Im} K)$ never vanishes and the proof of statement (d) is complete. 
Let $G=G_{R, \varepsilon} \subset D$ be a simple connected region with $\partial G=C$, where $C=C_{R, \varepsilon}:=C_{\varepsilon}^{1} \cup C_{\varepsilon}^{2} \cup C_{R} \cup L_{ \pm}^{1}(R, \varepsilon) \cup L_{ \pm}^{2}(R, \varepsilon) ; C_{\varepsilon}^{1}:=\{|k-1|=\varepsilon \ll 1\} ;$ $C_{\varepsilon}^{2}:=\{|k+1|=\varepsilon \ll 1\} ; C_{R}:=\{|k|=R \gg 1\} ;$ and $L_{ \pm}^{i}(R, \varepsilon)=L_{ \pm}^{i} \cap\{\varepsilon \leq$ $|k| \leq R\}$, see Figure 2 .

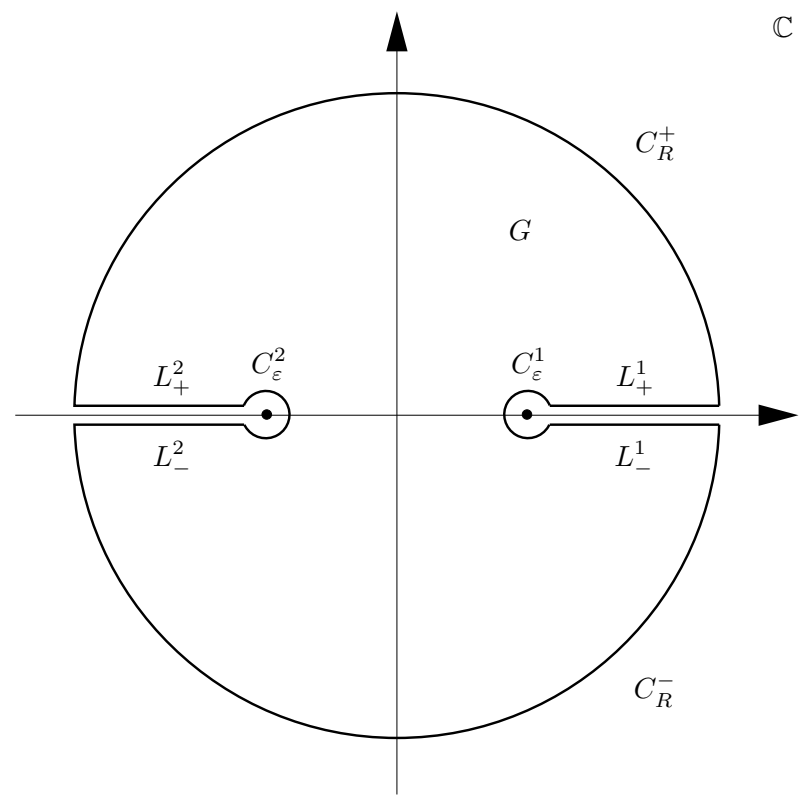

Figure 2. Domain $G_{R, \varepsilon}=G \subset D$.

Lemma 5. The elliptic functions $K$ and $E$ have no zeros in the domain $D \subset \mathbb{C}$.

Proof. In order to see that $E$ and $K$ do not vanish in $D$ we apply the Argument Principle to $G=G_{R, \varepsilon}$ for $R$ and $1 / \varepsilon$ positive and big enough.

We shall prove that the rotation number of $E$, when $k$ turns around the boundary of $G$ is less than 4 .

By Lemma 4.(b) the number of complete turns around $C_{R}$ is at most $1+\mu(R)$, where $\mu(R)$ tends to zero as $R$ goes to infinity. By Lemma 4.(c), since $\operatorname{Im} E \neq 0$ on $L_{ \pm}^{i}$, the number of complete turns on $L_{ \pm}^{1}(R, \varepsilon) \cup L_{ \pm}^{2}(R, \varepsilon)$ is less than 2 (in fact less than one half turn in each bank). Finally, by Lemma 4.(a), the number of complete turns of $E$ on $C_{\varepsilon}^{1} \cup C_{\varepsilon}^{2}$ when $\varepsilon$ goes to 0 tends to zero. Therefore, all together gives that the rotation number of $E$ when $k$ turns one time around the boundary of $G$ is less than 4 .

The study of $K$ is similar and gives the same result.

On the other hand, $E(i k), K(i k)>0, \quad$ for $k \in \mathbb{R}, \quad E(k), K(k)>0$, for $k \in(-1,1)$, 
and

$$
E(-k)=E(k), \quad E(\bar{k})=\bar{E}(k), \quad K(-k)=K(k), \quad K(\bar{k})=\bar{K}(k) .
$$

So if $E$ (or $K$ ) has a zero $z$ in $D$ it must have at least 4 zeros $\pm z, \pm \bar{z}$ in $D$, which is in contradiction with the Argument Principle. Hence the proof of this lemma is ended.

In the sequel we will use the notation $\#\{k \in A \mid f(k)=0\}=\#\{k \in$ $A \mid f=0\}$ to indicate the number of zeros of the function $f$ in the set $A$ taking into account their multiplicities.

Proof of Theorem 1. We begin by proving that $n+m+2$ is an upper bound for the number of zeros of $f K+g E$. As a first step we show how the case in which $f$ and $g$ have a non-constant common factor can be reduced to the case $\operatorname{gcd}(f, g)=1$. Assume that $h=\operatorname{gcd}(f, g)$, and that it has degree $d$. Then the equality

$$
f K+g E=h\left(\frac{f}{h} K+\frac{g}{h} E\right)
$$

implies that

$$
\begin{aligned}
& \#\{k \in D \mid f K+g E=0\} \\
& \leq \#\{k \in D \mid h(k)=0\}+\#\left\{k \in D \mid \frac{f}{h} K+\frac{g}{h} E=0\right\} .
\end{aligned}
$$

Since $\operatorname{gcd}(f / h, g / h)=1$, by assuming the theorem to be true in this case, we have

$$
\#\{k \in D \mid f K+g E=0\} \leq d+((n-d)+(m-d)+2) \leq n+m+2,
$$

as we wanted to prove.

So from now on we just consider the case $\operatorname{gcd}(f, g)=1$.

In our proof we consider two cases: $n \geq m+2$ and $n<m+2$.

Case $n \geq m+2$. By Lemma 5 ,

$$
\#\{k \in D \mid M(k)=0\}=\#\left\{k \in D \mid \bar{P}(k)=f+g \frac{E}{K}=0\right\} .
$$

As in the proof of Lemma 5 we apply the Argument Principle to $\bar{P}$ in $G=G_{R, \varepsilon}$ for $R$ and $1 / \varepsilon$ big enough.

By Lemma 4.(b) the number of complete turns around $C_{R}$ is at most $n+\mu(R)$, where $\mu(R)$ tends to 0 as $R$ goes to infinity.

By Lemma 4.(c)

$$
\begin{aligned}
\#\left\{k \in L_{ \pm}^{i} \mid \operatorname{Im} \bar{P}(k)=0\right\} & =\#\left\{k \in L_{ \pm}^{i} \mid g \operatorname{Im} \frac{E}{K}=0\right\} \\
& =\#\left\{k \in L_{ \pm}^{i} \mid g=0\right\}
\end{aligned}
$$


and hence the number of complete turns on $L_{ \pm}^{1}(R, \varepsilon) \cup L^{2}(R, \varepsilon)$ is less than $m+2$. Note that we have used that $\operatorname{gcd}(f, g)=1$ to ensure that $\bar{P}$ is not zero on the banks.

Finally, by Lemma 4.(a) the number of complete turns of $\bar{P}$ on $C_{\varepsilon}^{1} \cup C_{\varepsilon}^{2}$ when $\varepsilon$ goes to 0 tends to zero.

Therefore the rotation number of $\bar{P}(k)$ on $\partial G$ is at most $n+m+2$ and by the Argument Principle,

$$
\#\{k \in D \mid \bar{P}(k)=0\} \leq m+n+2,
$$

as we wanted to see.

The case $n<m+2$ follows by similar considerations by taking $g+f \frac{K}{E}$ instead of $f+g \frac{E}{K}$. Hence the first part of Theorem 1 is proved.

Next we prove the remaining three statements in the theorem.

(a). For any given $k_{i} \in(-1,1), i=1,2, \ldots, n+m+1$, consider the system of linear equations

$$
\sum_{j=0}^{n} a_{j} k_{i}^{j} K\left(k_{i}\right)+\sum_{j=0}^{m} b_{j} k_{i}^{j} E\left(k_{i}\right)=0, \quad i=1,2, \ldots, n+m+1 .
$$

Since the number of unknown variables $\left\{a_{j}\right\},\left\{b_{j}\right\}$ is greater than the number of equations, there exists a solution of $(9)$

$$
\left\{a_{j}\right\}_{j=0,1, \ldots, n}, \quad\left\{b_{j}\right\}_{j=0,1, \ldots, m} \text { with } \sum_{j=0}^{n} a_{j}^{2}+\sum_{j=0}^{m} b_{j}^{2}>0 .
$$

On the other hand, since by Lemma 4.(a), $K / E$ is not a rational function, we have that

$$
M(k)=\left(\sum_{i=0}^{n} a_{i} k^{i}\right) K+\left(\sum_{i=0}^{m} b_{i} k^{i}\right) E \not \equiv 0,
$$

and

$$
M\left(k_{i}\right)=0, \quad i=1,2, \ldots, n+m+1,
$$

as we wanted to see.

(b). By arguing as in statement (a) but with the function $f(k) K(\sqrt{k})+$ $g(k) E(\sqrt{k})$ and taking $k_{i}$ values in $(0,1), i=1,2 \ldots, n+m+1$, we have a function with $f$ and $g$ of degrees $n$ and $m$ respectively, and $n+m+1$ positive zeros. Hence the function $f\left(k^{2}\right) K(k)+g\left(k^{2}\right) E(k)$ is an even function with $2 n+2 m+2$ zeros (the values $\left.\pm \sqrt{k_{i}}\right)$ and $f\left(k^{2}\right)$ and $g\left(k^{2}\right)$ polynomials of degrees $2 n$ and $2 m$, respectively. Therefore the upper bound $n+m+2$ is attained as we wanted to see.

(c). Our proof is divided in two cases: 
(c.1) If $f$ has zeros in $[-1,1]$, then arguing as in the proof of the general upper bound we have that the complete turns of $\bar{P}$ on $L_{ \pm}^{1}(R, \varepsilon) \cup L_{ \pm}^{2}(R, \varepsilon)$ is at most $n+1$ (one less than if we have no information about $f$ ) and the result follows.

(c.2) If $f$ has no zeros in $[-1,1]$, then when $k \rightarrow \pm 1$ with $k \in(-1,1)$, the function $g+f \frac{K}{E}$ is real and tends to infinity with the same sign in both cases. Therefore the difference between the argument of $g+f \frac{K}{E}$ for $|k|<1$ near +1 and near -1 tends to be $2 L \pi$ for some integer number $L$. On the other hand arguing also as in the proof of the general upper bound but just taking the upper half part of the boundary of $G$ we obtain that this difference is smaller or equal than $(n+m+2) \pi$. Since $n+m$ is odd, in fact this difference has to be smaller than $(n+m+1) \pi$. By applying the same reasoning to the lower half boundary the result follows.

\section{Perturbation of an isochronous center.}

We need some preliminary results. In all this section $P_{i}, Q_{i}$ denote polynomials of degree $i$.

Lemma 6. Let $f$ be a continuous function and let $i, j \geq 0$ be integers. Then the following hold:

(a) If $i+j$ is odd, then

$$
\int_{0}^{2 \pi} f(\sin 2 \theta) \cos ^{i} \theta \sin ^{j} \theta d \theta=0 .
$$

(b) If $i+j=2 N$ even, then there exist real constants $C_{0}, C_{1}, \ldots, C_{N}$, such that

$$
\begin{aligned}
\int_{0}^{2 \pi} f(\sin 2 \theta) \cos ^{i} \theta \sin ^{j} \theta d \theta & =\sum_{s=0}^{N} C_{s} \int_{-\pi}^{\pi} f(\cos \theta) \cos ^{s} \theta d \theta \\
& =\sum_{s=0}^{N} C_{s} \int_{-\pi}^{\pi} f(\sin \theta) \sin ^{s} \theta d \theta .
\end{aligned}
$$

Proof. (a) Suppose that $i+j$ is odd. Then

$$
\begin{aligned}
I & =\int_{0}^{2 \pi} f(\sin 2 \theta) \cos ^{i} \theta \sin ^{j} \theta d \theta \quad(\theta=\pi+\varphi) \\
& =-\int_{0}^{2 \pi} f(\sin 2 \varphi) \cos ^{i} \varphi \sin ^{j} \varphi d \varphi \\
& =-I,
\end{aligned}
$$

which implies $I=0$. 
(b) Assume $i+j=2 N$ even. Then

$$
\begin{aligned}
I & =\int_{0}^{2 \pi} f(\sin 2 \theta) \cos ^{i} \theta \sin ^{j} \theta d \theta \quad\left(\theta=\frac{\pi}{4}-\varphi\right) \\
& =\int_{0}^{2 \pi} f(\cos 2 \varphi)\left(\frac{1}{\sqrt{2}} \cos \varphi+\frac{1}{\sqrt{2}} \sin \varphi\right)^{i}\left(\frac{1}{\sqrt{2}} \cos \varphi-\frac{1}{\sqrt{2}} \sin \varphi\right)^{j} d \varphi \\
& =\sum_{s=0}^{i+j} d_{s} \int_{-\pi}^{\pi} f(\cos 2 \varphi)(\cos \varphi)^{i+j-s}(\sin \varphi)^{s} d \varphi \\
& =\sum_{s=0}^{N} d_{2 s} \int_{-\pi}^{\pi} f(\cos 2 \varphi)(\cos \varphi)^{2 N-2 s}(\sin \varphi)^{2 s} d \varphi \\
& =\sum_{s=0}^{N} d_{2 s} \int_{-\pi}^{\pi} f(\cos 2 \varphi)\left(\frac{1+\cos 2 \varphi}{2}\right)^{N-s}\left(\frac{1-\cos 2 \varphi}{2}\right)^{s} d \varphi \\
& =\sum_{s=0}^{N} C_{s} \int_{-\pi}^{\pi} f(\cos 2 \varphi)(\cos 2 \varphi)^{s} d \varphi \quad(2 \varphi=\theta) \\
& =\sum_{s=0}^{N} C_{s} \int_{-\pi}^{\pi} f(\cos \theta)(\cos \theta)^{s} d \theta \quad\left(\theta=\frac{\pi}{2}-\varphi\right) \\
& =\sum_{s=0}^{N} C_{s} \int_{-\pi}^{\pi} f(\sin \varphi)(\sin \varphi)^{s} d \varphi, \quad
\end{aligned}
$$

where the value of the constants might vary from one expression to the other. Hence the proof of the lemma is complete.

\section{Lemma 7. Consider}

$$
J_{m}=J_{m}(h):=\int_{-\pi}^{\pi}(\sin \theta)^{2 m} \sqrt{h^{2} \sin ^{2} \theta+h} d \theta
$$

where $m$ is zero, or a natural number. Then there exist polynomials $P_{m}$ and $Q_{m}$ of degree $m$, such that

$$
J_{m}=\frac{k^{1-2 m}}{1-k^{2}}\left(P_{m}\left(k^{2}\right) K+Q_{m}\left(k^{2}\right) E\right)
$$

where $k^{2}=h /(1+h)$. 
Proof. We consider

(10)

$$
\begin{aligned}
J_{m} & =\int_{-\pi}^{\pi}(\sin \theta)^{2 m} \sqrt{h^{2} \sin ^{2} \theta+h} d \theta \\
& =\int_{-\pi}^{\pi}(\cos \theta)^{2 m} \sqrt{h^{2} \cos ^{2} \theta+h} d \theta \\
& =\int_{-\pi}^{\pi}\left(1-\sin ^{2} \theta\right)^{m} \sqrt{h^{2}+h-h^{2} \sin ^{2} \theta} d \theta \\
& =\sqrt{h^{2}+h} \int_{-\pi}^{\pi}\left(1-\sin ^{2} \theta\right)^{m} \sqrt{1-\frac{h}{h+1} \sin ^{2} \theta} d \theta \quad\left(k^{2}=\frac{h}{1+h}\right) \\
& =\frac{k}{1-k^{2}} \int_{-\pi}^{\pi} \sum_{i=0}^{m}(-1)^{i} C_{m}^{m-i}(\sin \theta)^{2 i} \sqrt{1-k^{2} \sin ^{2} \theta} d \theta \\
& =\frac{k}{1-k^{2}} \sum_{i=0}^{m}(-1)^{i} C_{m}^{m-i} B_{i},
\end{aligned}
$$

where

$$
B_{i}=\int_{-\pi}^{\pi}(\sin \theta)^{2 i} \sqrt{1-k^{2} \sin ^{2} \theta} d \theta
$$

If $\sin \theta=t$, then

$$
\begin{aligned}
B_{i} & =4 \int_{0}^{1} t^{2 i} \sqrt{\frac{1-k^{2} t^{2}}{1-t^{2}}} d t \\
& =4\left(O_{2 i}-k^{2} O_{2 i+2}\right),
\end{aligned}
$$

where

$$
O_{2 i}=\int_{0}^{1} \frac{t^{2 i}}{\sqrt{\left(1-t^{2}\right)\left(1-k^{2} t^{2}\right)}} d t .
$$

By formula 320.05 of $[\mathbf{2}], O_{2 i}$ satisfy the following recurrence formula:

$$
O_{2 i+2}=\frac{2 i\left(1+k^{2}\right) O_{2 i}+(1-2 i) O_{2 i-2}}{(2 i+1) k^{2}},
$$

and

$$
O_{0}=K, \quad O_{2}=\frac{1}{k^{2}}(K-E) .
$$

Thus, by induction we get

$$
B_{i}=P_{i}\left(\frac{1}{k^{2}}\right) K+Q_{i}\left(\frac{1}{k^{2}}\right) E,
$$


and hence substituting (11) into (10), we get

$$
J_{m}=\frac{k}{1-k^{2}}\left(P_{m}\left(\frac{1}{k^{2}}\right) K+Q_{m}\left(\frac{1}{k^{2}}\right) E\right) .
$$

From the above expression the lemma follows easily.

We also need the following result which has a straightforward proof.

Lemma 8. Consider

$$
W_{s}=W_{s}(h):=\int_{-\pi}^{\pi} \log \left(h \sin \theta+\sqrt{h^{2} \sin ^{2} \theta+h}\right)(\sin \theta)^{s} d \theta,
$$

where $s$ is zero, or a natural number. Then

$$
\frac{\partial W_{s}(h)}{\partial h}= \begin{cases}\frac{1}{2 h} \int_{-\pi}^{\pi} \sin ^{s}(\theta) d \theta, & \text { if } s \text { is even, } \\ \frac{1}{h}\left(J_{\frac{s+1}{2}}(h)-h \frac{\partial J_{\frac{s+1}{2}}(h)}{\partial h}\right), & \text { if } s \text { is odd, }\end{cases}
$$

where $J_{m}$ are defined in Lemma 7.

Lemma 9. Let $f(x), g(x)$ be analytic functions on $(a, b) \subset \mathbb{R}$, then

$$
\begin{aligned}
& \#\{x \in(a, b) \mid f(x)+g(x)=0\} \\
& \quad \leq \#\{x \in(a, b) \mid f(x)=0\}+\#\left\{x \in(a, b) \mid f g^{\prime}-g f^{\prime}=0\right\}+1 .
\end{aligned}
$$

Proof. Set $F=f+g$. Then

$$
F^{\prime} f-F f^{\prime}=f g^{\prime}-g f^{\prime} .
$$

We just make the proof for the case of simple zeros of $F$. The case of multiple zeros follows in a similar way. Let $x_{1}<x_{2}$ be two consecutive simple zeros of $F$. If $f$ does not vanish in $\left[x_{1}, x_{2}\right]$ then $F^{\prime} f$ has different signs in $x_{1}$ and $x_{2}$. Therefore the above expression implies that $f g^{\prime}-g f^{\prime}$ has a zero in $\left(x_{1}, x_{2}\right)$. Hence the lemma follows.

Proof of Theorem 2. Consider the polynomial perturbation the isochronous system $\dot{z}=i z+z^{3}$ :

$$
\begin{aligned}
& \dot{x}=-y+x^{3}-3 x y^{2}+\varepsilon P(x, y), \\
& \dot{y}=x+3 x^{2} y-y^{3}+\varepsilon Q(x, y),
\end{aligned}
$$

where $P, Q$ are real polynomials of degree $n$. For $\varepsilon=0,(13)$ has a first integral $(1+4 x y)\left(x^{2}+y^{2}\right)^{-2}$ with integrating factor $\left(x^{2}+y^{2}\right)^{-3} / 4$.

Denote by $\Gamma_{h}: H=h^{-1}(h>0)$ all periodic orbits surrounding the center $(0,0)$. In polar coordinates $x=r \cos \theta, y=r \sin \theta$,

$$
\Gamma_{h}: r=r_{h}(\theta)=r(\theta)=\sqrt{h \sin 2 \theta+\sqrt{h^{2} \sin ^{2} 2 \theta+h}} .
$$


By using (2) we know that the Abelian integral associated to (13) is defined as

$$
M(h)=\int_{\Gamma_{h}} \frac{P}{4\left(x^{2}+y^{2}\right)^{3}} d y-\frac{Q}{4\left(x^{2}+y^{2}\right)^{3}} d x .
$$

Denote by $D_{h}$ the simple connected region enclosed by $\Gamma_{h}$ and $D_{h, \delta}=D_{h} \backslash$ $\{r \leq \delta\}$.

By Green's formula

$$
M(h)=\iint_{D_{h, \delta}}\left[\frac{\partial P / \partial x+\partial Q / \partial y}{4\left(x^{2}+y^{2}\right)^{3}}-\frac{3 x P+3 y Q}{2\left(x^{2}+y^{2}\right)^{4}}\right] d x d y-T_{\delta},
$$

where

$$
T_{\delta}=\int_{r=\delta} \frac{P}{\left(x^{2}+y^{2}\right)^{3}} d y-\frac{Q}{\left(x^{2}+y^{2}\right)^{3}} d x .
$$

Let $\frac{1}{4}\left[\left(x^{2}+y^{2}\right)(\partial P / \partial x+\partial Q / \partial y)-6 x P-6 y Q\right]=\sum_{1 \leq i+j \leq n+1} C_{i, j} x^{i} y^{j}$. Then

$$
\begin{aligned}
M(h)= & \sum_{1 \leq i+j \leq n+1} C_{i, j} \iint_{D_{h, \delta}} \frac{x^{i} y^{j}}{\left(x^{2}+y^{2}\right)^{4}} d x d y-T_{\delta} \\
= & \sum_{1 \leq i+j \leq n+1} C_{i, j} \int_{0}^{2 \pi} \int_{\delta}^{r(\theta)} r^{i+j-7} \cos ^{i} \theta \sin ^{j} \theta d r d \theta-T_{\delta} \\
= & \sum_{\substack{i+j \neq 6 \\
1 \leq i+j \leq n+1}} C_{i, j} \int_{0}^{2 \pi} \frac{1}{i+j-6} r(\theta)^{i+j-6} \cos ^{i} \theta \sin ^{j} \theta d \theta \\
& +\sum_{i+j=6} C_{i, j} \int_{0}^{2 \pi} \log (r(\theta)) \cos ^{i} \theta \sin ^{j} \theta d \theta-C_{\delta},
\end{aligned}
$$

where

$$
\begin{aligned}
C_{\delta}= & \sum_{\substack{i+j \neq 6 \\
1 \leq i+j \leq n+1}} \frac{C_{i, j} \delta^{i+j-6}}{i+j-6} \int_{0}^{2 \pi} \cos ^{i} \theta \sin ^{j} \theta d \theta \\
& +\sum_{i+j=6} C_{i, j} \log \delta \int_{0}^{2 \pi} \cos ^{i} \theta \sin ^{j} \theta d \theta+T_{\delta} .
\end{aligned}
$$

We want to control the number of positive zeros of $M(h)$. In fact, in the final step we will study $\partial M(h) / \partial h$. Since $C_{\delta}$ does not depend on $h$ we do not take care of this constant. 
From the above formulas

$$
M(h)=\sum_{1 \leq i+j \leq n+1} C_{i, j} I_{i, j}-C_{\delta}
$$

where

$$
\begin{aligned}
& I_{i, j}=\frac{1}{i+j-6} \int_{0}^{2 \pi} r(\theta)^{i+j-6} \cos ^{i} \theta \sin ^{j} \theta d \theta, \quad i+j \neq 6, \\
& I_{i, j}=\int_{0}^{2 \pi} \log (r(\theta)) \cos ^{i} \theta \sin ^{j} \theta d \theta, \quad i+j=6 .
\end{aligned}
$$

By Lemma 6, we have that

$$
I_{i, j}=0, \quad \text { if } \quad i+j \quad \text { odd }
$$

and that there exist constants $C_{s}=C_{s}(i, j), s=0,1, \ldots, N$, such that $I_{i, j}$ is equal to

$$
\begin{cases}\sum_{s=0}^{N} C_{s} \int_{-\pi}^{\pi}(\sin \theta)^{s}\left(h \sin \theta+\sqrt{h^{2} \sin ^{2} \theta+h}\right)^{N-3} d \theta, & \text { if } i+j=2 N \neq 6, \\ \sum_{s=0}^{3} C_{s} \int_{-\pi}^{\pi}(\sin \theta)^{s} \log \left(h \sin \theta+\sqrt{h^{2} \sin ^{2} \theta+h}\right) d \theta, & \text { if } i+j=6 .\end{cases}
$$

Thus,

$$
M(h)=\sum_{N=1}^{\left[\frac{n+1}{2}\right]} I_{N}-C_{\delta}
$$

where

$$
I_{N}=\sum_{i+j=2 N} C_{i, j} I_{i, j}
$$

We calculate some compact expressions for $I_{N}$, for $N<3, N=3$ and $N>3$, separately. 
For $i+j=2 N<6$, by Lemma 6 , we have

$$
\begin{aligned}
I_{i, j} & =\sum_{s=0}^{N} C_{s} \int_{-\pi}^{\pi}(\sin \theta)^{s}\left(\frac{1}{\sqrt{h^{2} \sin ^{2} \theta+h}+h \sin \theta}\right)^{3-N} d \theta \\
& =\sum_{s=0}^{N} C_{s} \int_{-\pi}^{\pi}(\sin \theta)^{s}\left(\frac{\sqrt{h^{2} \sin ^{2} \theta+h}-h \sin \theta}{h}\right)^{3-N} d \theta \\
& =h^{N-3} \sum_{s=0}^{N} C_{s} \int_{-\pi}^{\pi}(\sin \theta)^{s} \sum_{m=0}^{3-N} C_{3-N}^{m}(-h)^{m}(\sin \theta)^{m} \\
& \cdot\left(h^{2} \sin ^{2} \theta+h\right)^{\frac{3-N-m}{2}} d \theta \\
& =\left\{\begin{array}{l}
2 \pi C_{0}\left(1+\frac{1}{h}\right)-\frac{2 C_{1}}{h} J_{1}, \quad \text { if } N=1, \\
-C_{1} \pi+\frac{C_{0}}{h} J_{0}+\frac{C_{2}}{h} J_{1}, \quad \text { if } N=2 .
\end{array}\right.
\end{aligned}
$$

Next we study $I_{i, j}$ for $i+j=6$. By (16),

$$
I_{i, j}=\sum_{s=0}^{3} C_{s} W_{s}, \quad W_{s}=\int_{-\pi}^{\pi} \log \left(h \sin \theta+\sqrt{h^{2} \sin ^{2} \theta+h}\right)(\sin \theta)^{s} d \theta .
$$

By Lemma 8,

$$
\begin{aligned}
\frac{\partial W_{0}}{\partial h}=\frac{\pi}{h}, & \frac{\partial W_{1}}{\partial h}=\frac{1}{h}\left(J_{1}-h \frac{\partial J_{1}}{\partial h}\right), \\
\frac{\partial W_{2}}{\partial h}=\frac{\pi}{2 h}, & \frac{\partial W_{3}}{\partial h}=\frac{1}{h}\left(J_{2}-h \frac{\partial J_{2}}{\partial h}\right) .
\end{aligned}
$$

Hence, we have

$$
\frac{\partial I_{3}}{\partial h}=\frac{d_{0}}{h}+d_{1} \frac{J_{1}}{h}+d_{2} \frac{J_{2}}{h}+d_{3} \frac{\partial J_{1}}{\partial h}+d_{4} \frac{\partial J_{2}}{\partial h},
$$

where $d_{i}$ are constants. 
For $i+j=2 N>6$,

(19)

$$
\begin{aligned}
I_{i, j}= & \sum_{s=0}^{N} C_{s} \int_{-\pi}^{\pi}(\sin \theta)^{s} \sum_{m=0}^{N-3} C_{N-3}^{m} h^{m}(\sin \theta)^{m}\left(h^{2} \sin ^{2} \theta+h\right)^{\frac{N-3-m}{2}} d \theta \\
= & \left\{\begin{array}{c}
h^{\frac{N-3}{2}} \sum_{s=0}^{N} C_{s} \int_{-\pi}^{\pi}(\sin \theta)^{s}\left(\sum_{m=0}^{\frac{N-3}{2}} p_{m}(\sin \theta)^{2 m} h^{m}\right. \\
\left.\quad+\sum_{m=0}^{\frac{N-5}{2}} q_{m}(\sin \theta)^{2 m+1} h^{m} \sqrt{h^{2} \sin ^{2} \theta+h}\right) d \theta, \text { if } N \text { odd } \\
h^{\frac{N}{2}-2} \sum_{s=0}^{N} C_{s} \int_{-\pi}^{\pi}(\sin \theta)^{s}\left(\sum_{m=0}^{\frac{N-4}{2}} p_{m}(\sin \theta)^{2 m+1} h^{m+1}\right. \\
\left.\quad+\sum_{m=0}^{\frac{N-4}{2}} q_{m}(\sin \theta)^{2 m} h^{m} \sqrt{h^{2} \sin ^{2} \theta+h}\right) d \theta, \text { if } N \text { even },
\end{array}\right.
\end{aligned}
$$

where $p_{m}, q_{m}$ are constants and we just have separated the odd and even values of $m$.

Remember that

$$
J_{m}=\int_{-\pi}^{\pi}(\sin \theta)^{2 m} \sqrt{h^{2} \sin ^{2} \theta+h} d \theta .
$$

From (19), we have

$$
I_{i, j}= \begin{cases}h^{\frac{N-3}{2}}\left(P_{\frac{N-3}{2}}(h)+\sum_{m=1}^{N-2} U_{m}(h) J_{m}\right), & \text { if } N \text { odd } \\ h^{\frac{N}{2}-2}\left(P_{\frac{N-2}{2}}(h)+\sum_{m=0}^{N-2} V_{m}(h) J_{m}\right), & \text { if } N \text { even }\end{cases}
$$

where $P_{\frac{N-3}{2}}, P_{\frac{N-2}{2}}, U_{m}, V_{m}$ are polynomials of $h$ with

$$
\begin{aligned}
& \operatorname{deg} P_{\frac{N-3}{2}} \leq \frac{N-3}{2}, \quad \operatorname{deg} P_{\frac{N-2}{2}} \leq \frac{N-2}{2} \\
& \operatorname{deg} U_{m} \leq \min \left\{m-1, \frac{N-5}{2}\right\}, \quad \operatorname{deg} V_{m} \leq \min \left\{m, \frac{N-4}{2}\right\} .
\end{aligned}
$$

From now on we introduce the variable $k$, as $k^{2}=h /(1+h)$. By Lemma 7 , equality (17) writes as

$$
\begin{aligned}
& I_{1}=\frac{1}{k^{2}}\left(P_{0}+k P_{1}\left(\frac{1}{k^{2}}\right) K+k Q_{1}\left(\frac{1}{k^{2}}\right) E\right), \\
& I_{2}=P_{0}+\frac{1}{k}\left(P_{1}\left(\frac{1}{k^{2}}\right) K+Q_{1}\left(\frac{1}{k^{2}}\right) E\right) .
\end{aligned}
$$


By using again Lemma 7, equality (20) writes as

$(22)$

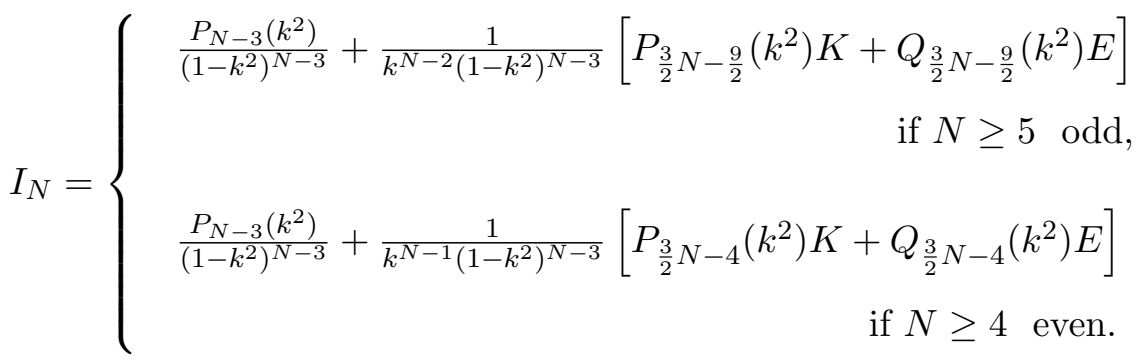

Let $N=\left[\frac{n+1}{2}\right]$, then for $n \geq 7$, we have that

$$
\begin{aligned}
& M(h)=\sum_{i=1}^{N} I_{i}-C_{\delta} \\
& \left\{\begin{array}{cc}
\frac{P_{1}\left(k^{2}\right)}{k^{2}}+I_{3}-C_{\delta}+\frac{P_{N-3}\left(k^{2}\right)}{\left(1-k^{2}\right)^{N-3}} & \\
+\frac{1}{k^{N-2}\left(1-k^{2}\right)^{N-3}}\left[P_{\frac{3}{2} N-\frac{9}{2}}\left(k^{2}\right) K+\right. & \text { if } N \geq 5 \quad \text { odd }, \\
\left.\frac{P_{1}\left(k^{2}\right)}{k^{2}}+I_{3}-C_{\delta}+\frac{P_{N-3}\left(k^{2}\right)}{\left(1-k^{2}\right)^{N-3}}\left(k^{2}\right) E\right], \\
+\frac{1}{k^{N-1}\left(1-k^{2}\right)^{N-3}}\left[P_{\frac{3}{2} N-4}\left(k^{2}\right) K+Q_{\frac{3}{2} N-4}\left(k^{2}\right) E\right], \\
& \text { if } N \geq 4 \quad \text { even. }
\end{array}\right.
\end{aligned}
$$

By (5), (18), taking into account that $d I_{3} / d k=2 k\left(1-k^{2}\right)^{-2}\left(d I_{3} / d h\right)$ and direct computations give that

$$
\frac{d M}{d k}= \begin{cases}k^{1-N}\left(1-k^{2}\right)^{2-N}\left(R_{0}+R_{1}\right), & \text { if } N \geq 5 \text { odd } \\ k^{-N}\left(1-k^{2}\right)^{2-N}\left(R_{0}+R_{1}\right), & \text { if } N \geq 4 \text { even }\end{cases}
$$

where

$$
\begin{aligned}
& R_{0}= \begin{cases}k^{N-4} P_{N-1}\left(k^{2}\right), & \text { if } N \geq 5 \text { odd, } \\
k^{N-3} P_{N-1}\left(k^{2}\right), & \text { if } N \geq 4 \text { even, }\end{cases} \\
& R_{1}= \begin{cases}P_{\frac{3}{2} N-\frac{7}{2}}\left(k^{2}\right) K+Q_{\frac{3}{2} N-\frac{7}{2}}\left(k^{2}\right) E, & \text { if } N \geq 5 \text { odd }, \\
P_{\frac{3}{2} N-3}\left(k^{2}\right) K+Q_{\frac{3}{2} N-3}\left(k^{2}\right) E, & \text { if } N \geq 4 \text { even. }\end{cases}
\end{aligned}
$$


Next we estimate the number of zeros of $d M / d k$ by applying Lemma 9 to $R_{0}+R_{1}$. Straightforward computations show that

$$
R_{0} R_{1}^{\prime}-R_{0}^{\prime} R_{1}= \begin{cases}\frac{k^{N-5}}{1-k^{2}} R, & \text { if } N \geq 5 \text { odd } \\ \frac{k^{N-4}}{1-k^{2}} R, & \text { if } N \geq 4 \text { even }\end{cases}
$$

where

$$
R= \begin{cases}P_{\frac{5}{2} N-\frac{7}{2}}\left(k^{2}\right) K+Q_{\frac{5}{2} N-\frac{7}{2}}\left(k^{2}\right) E, & \text { if } N \geq 5 \text { odd }, \\ P_{\frac{5}{2} N-3}\left(k^{2}\right) K+Q_{\frac{5}{2} N-3}\left(k^{2}\right) E, & \text { if } N \geq 4 \text { even. }\end{cases}
$$

By Theorem 1, we obtain

$$
\#\{-1<k<1 \mid R=0\} \leq \begin{cases}2(5 N-7)+2=10 N-12, & \text { if } N \geq 5 \text { odd, } \\ 2(5 N-6)+2=10 N-10, & \text { if } N \geq 4 \text { even. }\end{cases}
$$

Note that $R$ is an even function. Therefore we have

$$
\#\{0<k<1 \mid R=0\} \leq \begin{cases}5 N-6, & \text { if } N \geq 5 \text { odd } \\ 5 N-5, & \text { if } N \geq 4 \text { even. }\end{cases}
$$

By Lemma 9 we obtain

$$
\begin{aligned}
& \#\left\{0<k<1 \mid \frac{d M}{d k}=0\right\} \\
& \leq \begin{cases}N-1+5 N-6+1=6 N-6, & \text { if } N \geq 5 \text { odd, } \\
N-1+5 N-5+1=6 N-5, & \text { if } N \geq 4 \text { even. }\end{cases}
\end{aligned}
$$

From Rolle's Theorem, it follows that

$$
\begin{aligned}
& \#\{h>0 \mid M(h)=0\}=\#\{0<k<1 \mid M(k)=0\} \\
& \leq \begin{cases}6 N-5=6\left[\frac{n+1}{2}\right]-5, & \text { if }\left[\frac{n+1}{2}\right] \geq 5 \quad \text { odd, } \\
6 N-4=6\left[\frac{n+1}{2}\right]-4, & \text { if }\left[\frac{n+1}{2}\right] \geq 4 \text { even, }\end{cases} \\
& \leq 3 n-1 .
\end{aligned}
$$

From the above inequality the theorem follows.

Acknowledgement. Weigu $\mathrm{Li}$ and Zhifen Zhang want to thank to the CRM and to the Department of Mathematics of the Universitat Autònoma de Barcelona for their support and hospitality during the period in which this paper was written. 


\section{References}

[1] T.R. Blows and L.M. Perko, Bifurcation of limit cycles from centers and separatrix cycles of planar analytic systems, SIAM Rev., 36 (1994), 341-376, MR 95g:58155, Zbl 0807.34051.

[2] P.F. Byrd and M.D. Friedman, Handbook of Elliptic Integrals for Engineers and Physicists, Springer-Verlag, Berlin, 1954, MR 15,702a, Zbl 0055.11905.

[3] C. Chicone and M. Jacobs, Bifurcation of limit cycles from quadratic isochronous, J. Diff. Equations, 91 (1991), 268-326, MR 92e:58143, Zbl 0733.34045.

[4] E.A. Coddington and N. Levinson, Theory of Ordinary Differential Equations, McGraw-Hill Book Company, Inc., New York-Toronto-London, 1955, MR 16,1022b, Zbl 0064.33002.

[5] F. Dumortier, C. Li and Z. Zhang, Unfolding of a quadratic integrable system with two centers and two unbounded heteroclinic loops, J. Diff. Equations, 139 (1997), 146-193, MR 98f:34034, Zbl 0883.34035.

[6] H. Giacomini, J. Llibre and M. Viano, On the nonexistence, existence, and uniqueness of limit cycles, Nonlinearity, 9 (1996), 501-516, MR 97a:34073, Zbl 0886.58087.

[7] - On the shape of limit cycles that bifurcate from Hamiltonian centers, Nonlinear Anal., 41(3-4) (2000), 523-537, MR 2001e:34052, Zbl 0954.34022.

[8] _ The shape of limit cycles that bifurcate from non-Hamiltonian centers, Nonlinear Anal., 43(7) (2001), 837-859, MR 2001k:37077.

[9] I.D. Iliev, Perturbations of quadratic centers, Bull. Sci. Math., 122 (1998), 107-161, MR 99a:34082, Zbl 0920.34037.

[10] C. Li, W. Li, J. Llibre and Z. Zhang, On the limit cycles of polynomial differential systems with homogeneous nonlinearities, Proc. Edinburgh Math. Soc., 43 (2000), 529-543.

[11] Bifurcation of limit cycles from quadratic isochronous centers, Nonlinearity, 13 (2000), 1775-1800, MR 2001i:34050.

[12] L Linear estimate of the number of zeros of Abelian integrals for some cubic isochronous centers, to appear in J. Diff. Equations.

[13] J. Llibre, J.S. Pérez del Río and J.A. Rodríguez, Averaging analysis of a perturbed quadratic center, Nonlinear Analysis, Ser. A: Theory, Methods, 46 (2001), 45-51, CMP 1845576.

[14] G.S. Petrov Number of zeros of complete elliptic integrals, Funct. Anal. Appl., 18 (1984), 148-149, MR 85j:33002, Zbl 0567.14006.

[15] _ Elliptic integrals and their oscillation, Funct. Anal. Appl., 20 (1986), 37-40, MR 87f:58031, Zbl 0656.34017.

[16] _ The Chebyshev property of elliptic integrals, Funct. Anal. Appl., 22 (1988), 72-73, MR 89i:33002, Zbl 0653.33001.

[17] C. Rousseau, Bifurcation methods in polynomial systems, in 'Bifurcation and Periodic Orbits of Vector Fields', NATO ASI Series, Serie C: Math and Phys. Sciences, 408 (1993), 383-428; edited by D. Schlomiuk, MR 95a:34059, Zbl 0791.58080.

Received March 9, 2000 and revised June 13, 2000. A. Gasull and J. Llibre are partially supported by a DGES grant number PB96-1153 and by a CICYT grant number 1999SGR00349. 
Departament de MATEMÀtiques

Universitat Autònoma de Barcelona 08193 BellaterRA

BARCELONA

SPAIN

E-mail address: gasull@mat.uab.es

Departament de Matemàtiques

Universitat Autònoma de Barcelona 08193 Bellaterra

BARCELONA

SPAIN

Departament de Matemàtiques

Universitat Autònoma de Barcelona 08193 BeLlaterRA

BARCELONA

SPAIN

E-mail address: jllibre@mat.uab.es

Departament de Matemàtiques

Universitat Autònoma de Barcelona 08193 BeLlaterRA

BARCELONA

SPAIN

Department of Mathematics

Peking University

BEIJING 100871

CHINA

E-mail address: weigu@math.pku.edu.cn 